\title{
Cigarette smoking increases the risk of coronary heart disease in women more than in men
}

The risk of coronary heart disease (CHD) is $25 \%$ greater in women who smoke cigarettes than in male smokers, independent of other cardiovascular risk factors. This finding comes from a systematic review and meta-analysis of data from more than 2.4 million people worldwide. The authors of the report, published in the Lancet, believe that this figure could even be an underestimate and the actual female risk is higher; "the relative immaturity of the smoking epidemic among women compared with men, as well as the fact that women tend to smoke fewer cigarettes than men, are [likely to be] important in driving the underestimation", explains Dr Rachel Huxley.

The investigators conducted their review according to the Meta-analysis of Observational Studies in Epidemiology guidelines. Of the 8,005 articles (published between January 1966 and December 2010) identified in the database search, 17 reports (75 cohorts) were selected for inclusion in the analysis. A total of 43,995 CHD events occurred among the 2,409,955 individuals in these cohorts.

The pooled ratio of relative risk of smoking versus nonsmoking for females compared with males was 1.25 (95\% CI $1.12-1.39, P<0.0001)$. When the outcome was restricted to fatal CHD events, the excess risk for female smokers was $19 \%$ (95\% CI 1.06-1.34, $P=0.004$ ). Notably, the risk increased by $2 \%$ for every year of study follow-up. The findings did not differ between geographical regions (AsiaPacific compared with Europe and North America). Female smokers had a higher risk of CHD than men across all age groups, with the exception of ages 30-44 years, although the difference between the sexes was only significant for ages 60-69 years.

Why smoking is more harmful to women than men is not fully understood; however, the authors speculate that the absorption or pathophysiological effects of toxins from cigarette smoke might be greater

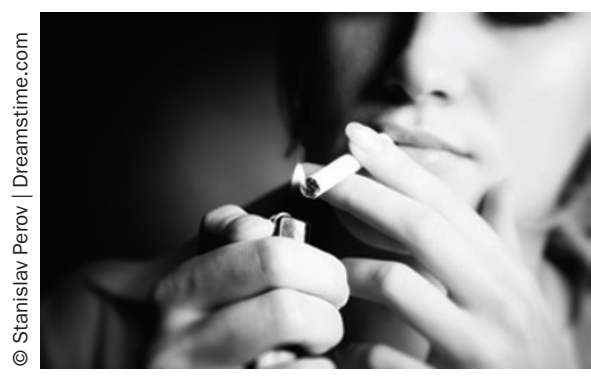

in women. They conclude their report by recommending that "physicians and health professionals should be encouraged to increase their efforts at promotion of smoking cessation ... [and] that inclusion of a female perspective in tobacco-control policies is crucial."

\section{Alexandra King}

\section{Original article Huxley, R. R. \& Woodward, M. Cigarette} smoking as a risk factor for coronary heart disease in women compared with men: a systematic review and meta-analysis of prospective cohort studies. Lancet doi:10.1016/ S0140-6736(11)61035-0 